\title{
Cryptococcosis and unexpected death
}

\author{
Abbie Tu ${ }^{1} \cdot$ Roger W. Byard ${ }^{1}[$ \\ Accepted: 3 June 2021 / Published online: 29 July 2021 \\ (c) Springer Science+Business Media, LLC, part of Springer Nature 2021
}

\begin{abstract}
Cryptococcosis is a fungal disease caused predominantly by Cryptococcus neoformans and Cryptococcus gatti. It is most often found in immunocompromised individuals and has quite protean and chronic manifestations affecting all body systems. The unexpected death of a 22-year-old man with cryptococcal meningoencephalitis demonstrates, however, that it may have a fulminant course in previously well individuals. Also present at autopsy was a toruloma of the upper lobe of the right lung. Delays in clinical diagnoses, confusion with tuberculosis and precipitate clinical deterioration may mean that cases will be encountered unexpectedly during medicolegal autopsies.
\end{abstract}

Keywords Cryptococcosis $\cdot$ Torulosis $\cdot$ Unexpected death $\cdot$ Forensic $\cdot$ Meningoencephalitis

\section{Case report}

As sudden and unexpected death is an unusual occurrence in cases of cryptococcosis in many forensic settings the following case involving an apparently immunocompetent man is reported from The University of Adelaide Pathology museum archive. Clinical manifestations and possible findings at autopsy are reviewed.

A 22-year-old man was admitted to hospital with a two and a half week history of generalized headache that worsened when he bent over or ran down stairs. Three days prior to admission he had commenced vomiting. On admission he had a mild fever with moderate neck stiffness but no localizing neurological signs. A chest $\mathrm{x}$-ray showed a discrete mass anterior to the hilum of the right lung and a lumbar puncture revealed numerous Cryptococcus neoformans organisms. Several days later he unexpectedly had a generalized fit and died on the ward. Although no further clinical information was available from the autopsy report he had apparently previously been quite well and working.

At autopsy the body was that of a thin adult male. The major findings were localized to the central nervous system. The brain was swollen with flattening of gyri, congestion and

Roger W. Byard

roger.byard@sa.gov.au

1 School of Anatomy and Pathology, University of Adelaide and Forensic Science SA, Adelaide, South Australia, Australia hemorrhage in the cerebellar tonsils, and focal subarachnoid opacity due to edema fluid (Fig. 1). Sectioning revealed collapse of both lateral ventricles with cystic areas within both basal ganglia, particularly on the left side in the head of the caudate nucleus and in the lentiform nucleus (Fig. 2). The posterior aspect of the upper thoracic spinal cord showed gelatinous discoloration. A mass was also present in the upper lobe of the right lung measuring $40 \times 50 \times 50 \mathrm{~mm}$. It had an irregular scalloped edge demarcated from the surrounding compressed lung with loose trabeculae separated by gelatinous parenchyma typical of a "toruloma", the nodular lesion of cryptococcosis (Fig. 3). Histological examination of sections from the central nervous system and lung mass revealed numerous encapsulated Cryptococcus neoformans organisms (Fig. 4). Death was therefore due to infection by Cryptococcus neoformans.

\section{Discussion}

Cryptococcosis is a potentially lethal infection most commonly caused by two fungi, Cryptococcus neoformans and Cryptococcus gatti. Cryptococcus neoformans, which is responsible for $80 \%$ of cases, can cause acute, subacute or chronic disease which most often involves the respiratory and central nervous systems [1]. This encapsulated yeastlike fungus was first isolated from peach juice in 1895, and then was subsequently found in milk, soil, and avian excrement, including pigeons, chickens, parrots, canaries, 


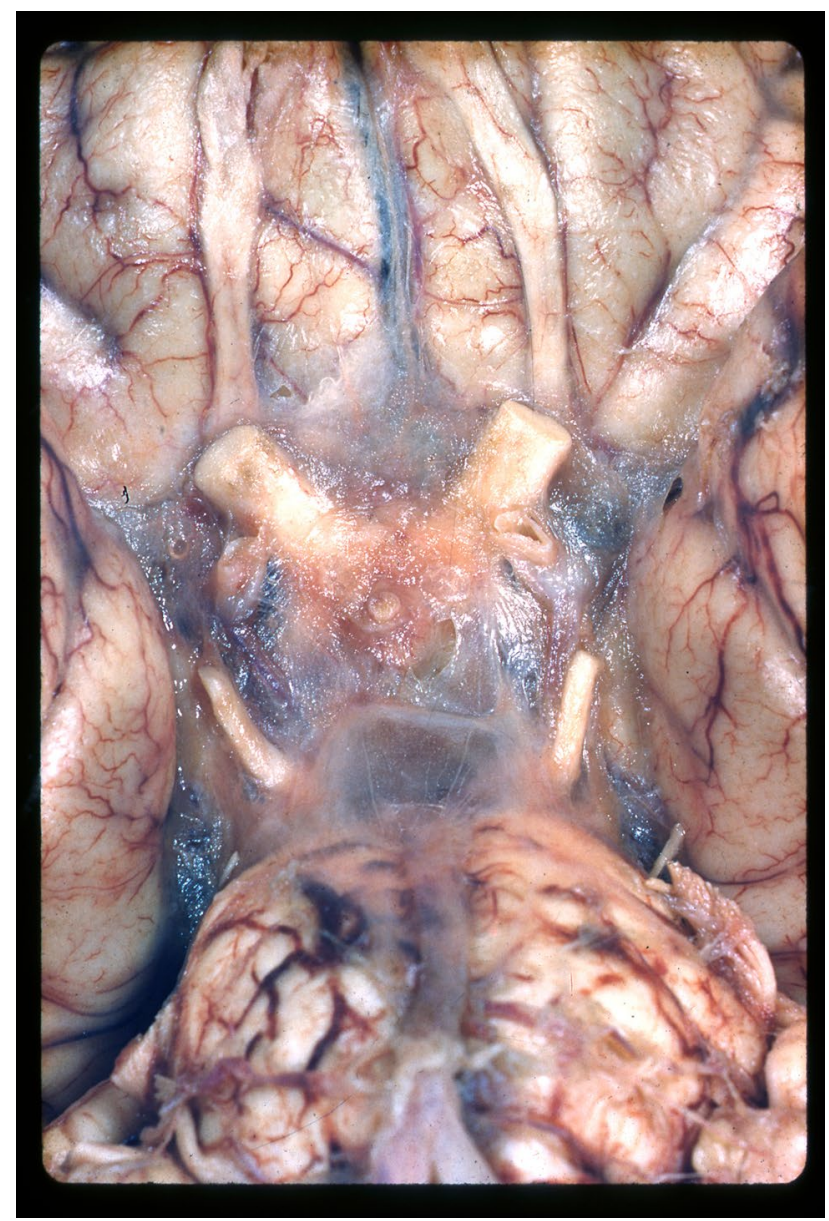

Fig. 1 Opacity of the subarachnoid space around the optic chiasm due to edema associated with Cryptococcus neoformans

sparrows, and skylarks [2]. The association between Cryptococcus neoformans and nervous system infection was first described by von Hansmann in 1905 [3].

Cryptococcus neoformans is transmitted to humans mainly by inhalation of airborne organisms from the environment. The spores or dried yeast cells will then accumulate and colonize the surface of the respiratory tract where they may remain dormant for years [1]. The primary stage of infection will usually, therefore, be found in the lungs with further spread to other organ systems following a hematogenous route [4].

Three distinct groups of at risk individuals were identified by the Infectious Diseases Society of America (IDSA): those who are HIV positive, immunosuppressed recipients of organ transplants and a final heterogeneous group of HIV-negative/non-transplant patients [5, 6]. Although immunocompromised individuals are more susceptible to cryptococcosis, paradoxically, non-immunocompromised individuals often have a more severe clinical course [1]. Cryptococcosis is not a rare disease globally with the highest

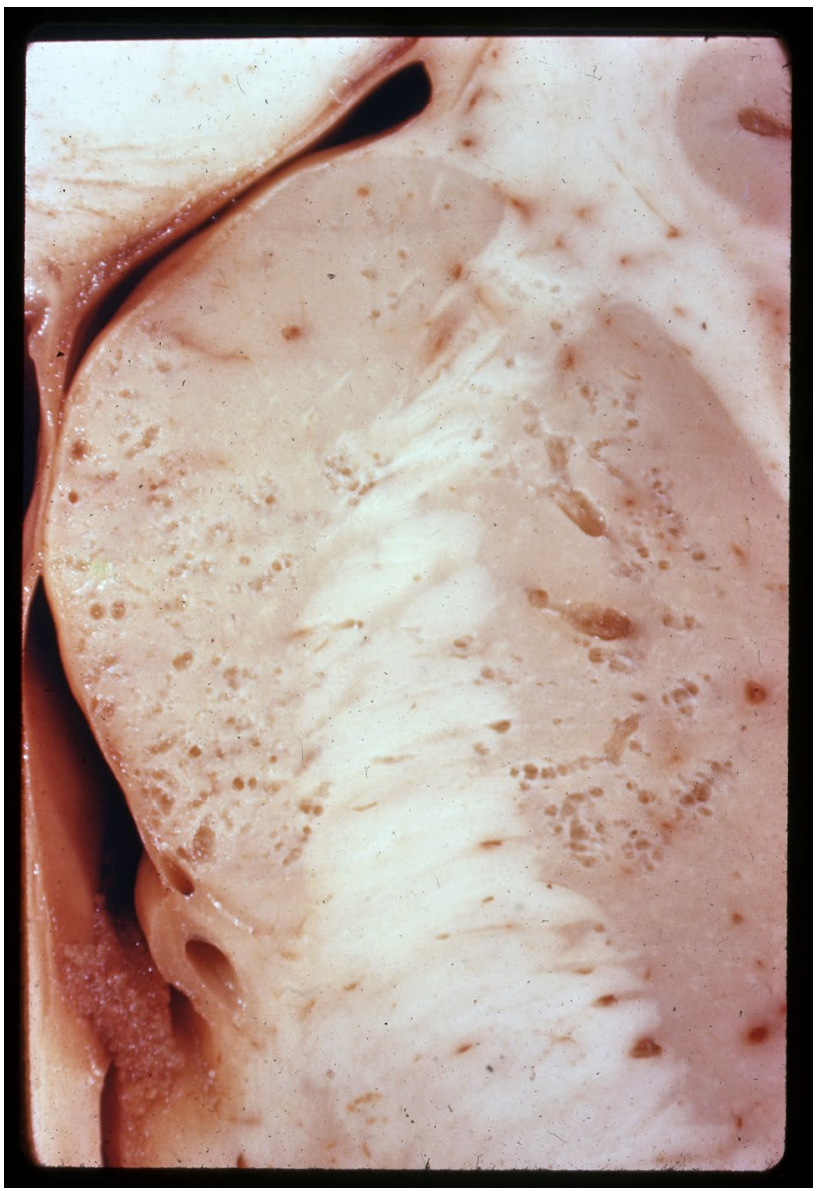

Fig. 2 Cystic changes within the basal ganglia on the left side due to Cryptococcus neoformans

number of deaths recorded each year in the first decade of this century being approximately 600,000 [7]. More than 1 million cases of Cryptococcus neoformans infections have been detected in AIDS patients annually [8]. Cryptococcal

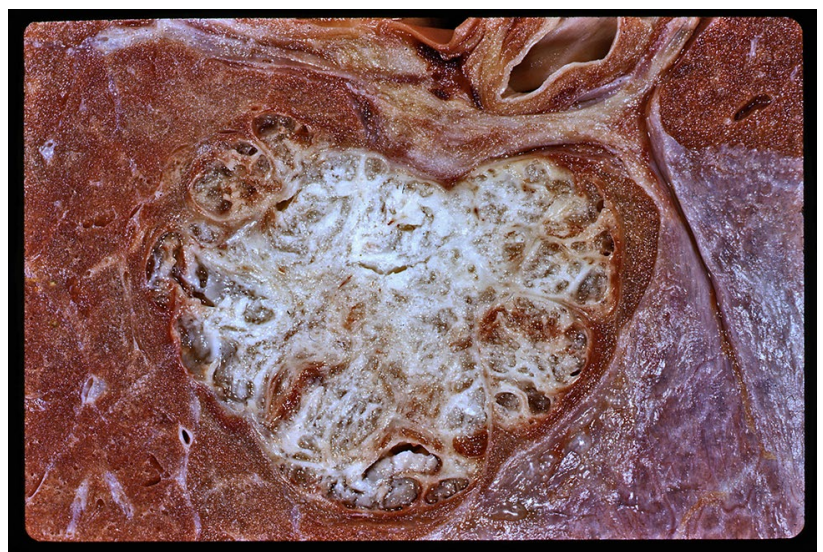

Fig. 3 A 'toruloma' of the upper lobe of the right lung showing fibrous trabeculae separated by gelatinous parenchyma caused by pulmonary infiltrated by Cryptococcus neoformans 


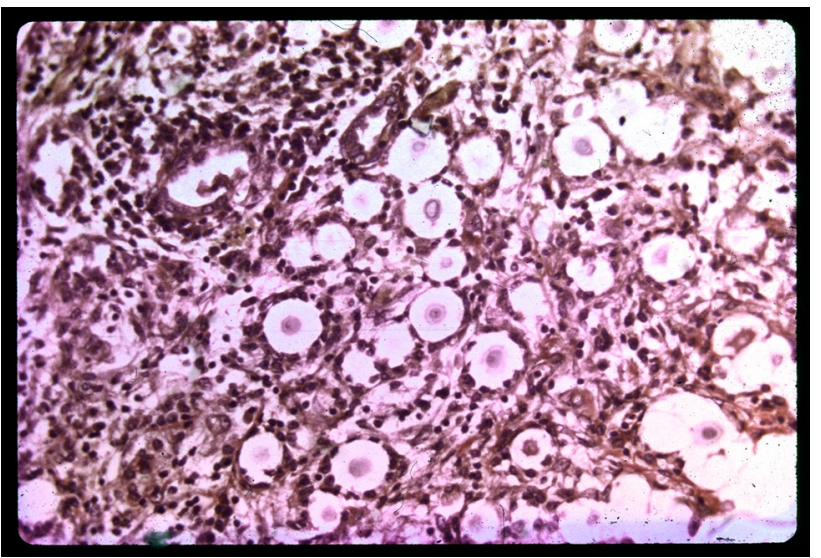

Fig. 4 Typical aggregates of encapsulated Cryptococcus neoformans in the lung (Hematoxylin and eosin $\times 150$ )

meningoencephalitis is associated with the highest mortality rate with approximately one million cases occurring globally per year [4].

There is a greater than $60 \%$ mortality rate in the first three months of infection with $80 \%$ of cases occurring in subSaharan Africa associated with HIV infection. The mortality remains high (15-30\%) despite appropriate treatment with amphotericin B and flucytosine [4, 9]. The high incidence of morbidity and mortality in semi-developed countries in sub-Saharan Africa and parts of Asia results from limited heath care resources and impoverished medical facilities.

The COVID-19 pandemic has focused attention on the potential for transmission of pathogens from animal reservoirs, and zoonotic transmission of Cryptococcus neoformans from birds to humans is also recognized. For example, Cryptococcus neoformans was transmitted from a pet cockatoo to a renal transplant recipient and from a magpie to an immunocompetent patient in 2000 and 2005 respectively. Cryptococcus neoformans was isolated from both the patients' cerebrospinal fluid and also from the magpie's excreta $[10,11]$.

Pulmonary cryptococcal infection is not necessarily symptomatic or may merely resemble a mild respiratory viral infection. Later stage disease may be associated with weight loss, cough, fever and chest pain. Immunocompromised patients are more likely to progress rapidly to pneumonia [8]. The mass in the lung of the reported cases demonstrates that the cryptoccocal infection had been present for some time in the decedent.

As can be seen from the reported case central nervous system infection can have dire consequences. Cryptococcus neoformans spreads via the circulation from the lungs and passes through the blood brain barrier by a variety of mechanisms including paracellular passage between endothelial cells, transcytosis through endothelial cells, or by infecting monocytes/macrophages, the so-called 'Trojan horse' effect [4]. Both Cryptococcus neoformans and Cryptococcus gatti exhibit a unique tropism for the brain [1]. Clinical effects include headache, hearing and visual loss, malaise, stroke and altered mental state. It has been suggested that the higher mortality rate from cryptococcal meningitis in the non-immunocompromised is due to delays in diagnosis [1]. Cerebrospinal fluid pressure is often raised and individuals without immunocompromise often present with obstructive hydrocephalus [9].

Cryptoccocal infection may rarely cause endocarditis of both native and prosthetic valves and may follow cardiac catheterization and valvotomy [12], and may occur in the immunocompetent [13]. Immunocompromised individuals with prosthetic valves are at higher risk of pericarditis and myocarditis [13].

Skeletal cryptococcosis infections are extremely rare and bone infections can be both acute and chronic arising from hematogenous dissemination from a primary pulmonary infection. Bone pain is a common presentation with the infected area resembling an abscess containing mucoid and gelatinous material [14]. Multiple sites of infection have been reported including the knee, humerus, scapula, rib and costochondral junction. Organisms may be also found disseminated throughout the body in lymph nodes, prostate, eye, skin, heart spleen, liver, kidneys and adrenal glands $[15,16]$.

Imaging studies vary in their effectiveness in diagnosing the disease depending on the infection site and severity. Single or more pulmonary nodules tend to be found in immunocompetent patients, as in the reported case, while immunosuppressed patients more frequently have reticular interstitial changes associated with cavitation, consolidation and pleural effusions $[8,9,17]$. CT scans in the brain are often non-specific for cryptococcal meningitis although MRI scans are more sensitive in assessing nodules in the meninges and cerebral parenchyma.

Polysaccharide antigen detection is used for the rapid diagnosis of cryptococcal disease diagnosis [18]. Antigen detection in cerebrospinal fluid is more sensitive than in the serum, particularly if there is pulmonary and meningeal cryptococcosis [15]. The lateral flow assay (LFA) provides a rapid and highly specific method for measuring cryptococcal antigen titres and is particularly useful for detecting cryptococcal meningitis as it involves a simple plasma test and can be used in patients who have low antigen levels. As latex agglutination and latera flow assay cannot differentiate live from dead organisms the assessment of treatment response is limited $[3,8]$.

Cultures provide an alternative laboratory diagnostic method with Cryptococcus neoformans growing best at 30 to $35{ }^{\circ} \mathrm{C}$ under aerobic conditions. However growth may take up to 4 weeks particularly if patients have had therapy [7]. The India ink stain is an alternative, cost effective, and rapid 
method for identifying cryptococcus that was described in the 1950s [16]. Although the India ink stain has a 50\% positivity for patients with suspected cryptococcal meningitis, false-positive results can occur and it also cannot differentiate live from dead fungal cells.

Thus, cryptococcosis is a potentially lethal infection that can affect multiple body systems with quite variable symptoms and signs. Delays in clinical diagnoses and confusion with tuberculosis mean that cases may present undiagnosed to forensic facilities and so an awareness of its protean manifestations is important. Although it is more common in the immunocompromised it may also rarely occur in previously well non-immunocompromised individuals with a fulminant course and on occasion rapid and unexpected death.

\section{References}

1. Levitz SM. The ecology of Cryptococcus neoformans and the epidemiology of cryptococcosis. Rev Infect Dis. 1991;13:1163-9.

2. Holmes SJ, Hawks GH. Torulosis of the central nervous system. Can Med Assoc J. 1953;68:143-6.

3. Anjum $\mathrm{S}$, Williamson PR. Clinical aspects of immune damage in cryptococcosis. Curr Fungal Infect Rep. 2019;13:99-108.

4. Sabiiti W, May RC. Mechanisms of infection by the human fungal pathogen Cryptococcus neoformans. Future Microbiol. 2012;7:1297-313.

5. Bratton EW, Husseini El N, Chastain CA, Lee MS, Poole C, Stürmer T, et al. Comparison and temporal trends of three groups with cryptococcosis: HIV-infected, solid organ transplant, and HIV-negative/non-transplant. PLOS One. 2012;7:e43582.

6. Perfect JR, Dismukes WE, Dromer F, Goldman DL, Graybill JR, Hamill RJ, et al. Clinical practice guidelines for the management of cryptococcal disease: 2010 update by the Infectious Diseases Society of America. Clin Infect Dis. 2010;50:291-322.
7. Maziarz EK, Perfect JR. Cryptococcosis. Infect Dis Clin North Am. 2016;30:179-206.

8. Skolnik K, Huston S, Mody CH. Cryptococcal lung infections. Clin Chest Med. 2017;38:451-64.

9. Bicanic T, Harrison TS. Cryptococcal meningitis. Br Med Bull. 2005;72:99-118.

10. Nosanchuk JD, Shoham S, Fries BC, Shapiro DS, Levitz SM, Casadevall A. Evidence of zoonotic transmission of Cryptococcus neoformans from a pet cockatoo to an immunocompromised patient. Ann Intern Med. 2000;132:205-8.

11. Lagrou K, Van Eldere J, Keuleers S, Hagen F, Merckx R, Verhaegen J, et al. Zoonotic transmission of Cryptococcus neoformans from a magpie to an immunocompetent patient. J Intern Med. 2005;257:385-8.

12. Banerjee U, Gupta K, Venugopal P. A case of prosthetic valve endocarditis caused by Cryptococcus neoformans var. neoformans. J Med Vet Mycol. 1997;35:139-41.

13. Roy M, Ahmad S, Roy AK. Cryptococcus neoformans infective endocarditis of native valves in an immunocompetent host. ID Cases. 2018;12:66-70.

14. Behrman RE, Masci JR, Nicholas P. Cryptococcal skeletal infections: case report and review. Rev Infect Dis. 1990;12:181-90.

15. Benešová $P$, Buchta V, Cerman J, Žák P. Cryptococcosis-a review of 13 autopsy cases from a 54-year period in a large hospital. APMIS. 2007;115:177-83.

16. Littman ML. Cryptococcosis (torulosis). Current concepts and therapy. Am J Med. 1959;27:976-98.

17. Chang WC, Tzao C, Hsu HH, Lee SC, Huang KL, Tung HJ, Chen CY. Pulmonary cryptococcosis: comparison of clinical and radiographic characteristics in immunocompetent and immunocompromised patients. Chest. 2006;129:333-40.

18. Abassi M, Boulware DR, Rhein J. Cryptococcal meningitis: diagnosis and management uptake. Curr Trop Med Rep. 2015;2:90-9.

Publisher's Note Springer Nature remains neutral with regard to jurisdictional claims in published maps and institutional affiliations. 